\title{
Immediate and early loading of tapered implants in extraction sites: 10-year retrospective follow-up
}

IMPLANT THERAPY OUTCOMES, PERI-IMPLANT BIOLOGY ASPECTS

\section{Priamo Mura}

Studio Priamo Mura, Rome, Italy

\section{Background}

In order to restore function to patients with missing or failing dentition as quickly and safely as possible, implants should be able to achieve primary stability and show good survival outcomes in clinical situations. ${ }^{1,2}$ This is particularly important when implants are placed in fresh extraction sites and with immediate loading protocols. ${ }^{3}$

\section{Aim}

This retrospective study evaluated 10 -year clinical and radiologic outcomes of patients treated with tapered implants in post-extraction sites using immediate or early loading protocols.

\section{Methods and Materials}

Patients were included who received at least one implant (Replace Select Tapered TiUnite, Nobel Biocare AB, Göteborg, Sweden) in a fresh extraction site with either an immediate ( $\leq 48$ hours) or early (2-19 days) loading protocol (Figure 1$)$.

- All treatments were performed with a flapless approach.

- Implants were followed at regular intervals for a minimum of 10 years.

- Radiographs taken at regular patient recalls were analyzed for marginal bone levels and bone remodeling.

- Implant stability and Jemt's papilla index ${ }^{4}(\mathrm{PI})$ were also analyzed.

- Descriptive statistical analysis of available data was performed with SPSS v23 (IBM, USA)

Figure 1 Replace Select Tapered TiUnite implant used in this study for replacement of teeth in fresh extraction sites.

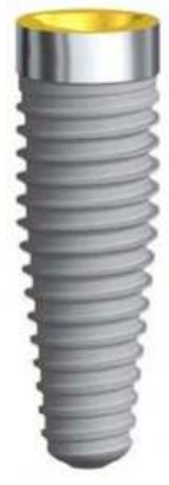

\section{Results}

- 61 patients ( 38 female, 23 male; mean age $50.3 \pm 14.3$ years) received 89 tapered implants in 85 fresh extraction sites ( 4 molar extraction sites received 2 implants each, Table 1).

79 implants $(88.8 \%)$ were loaded on the day of surgery. The remaining 10 implants were loaded $7-19$ days after insertion.

- The mean insertion torque was $44.8 \pm 3.6 \mathrm{Ncm}$ (range $40-50 \mathrm{Ncm}$ )

- Delivery of the definitive prosthesis occurred after a mean of $2.8 \pm 0.7$ months.

- 32 patients (49 implants) completed the 10-year follow-up (mean 11.6 \pm 0.6 years, range $10.4-12.8$ years)

Papilla height was favorable (PI score 2 or 3 ) for most implants at 5-year follow-up and $98 \%$ of the papillae evaluated at the 10 -year follow-up remained stable from the 5 -year results (Figure 2).

- The mean marginal bone level at baseline was $-1.80 \pm 2.07 \mathrm{~mm}(\mathrm{n}=89)$ and $-2.64 \pm 1.30 \mathrm{~mm}(\mathrm{n}=49)$ at 10-year follow-up.

- The mean marginal bone remodelling between baseline and 10-year follow-up was $-0.86 \pm 2.10 \mathrm{~s} \mathrm{~mm}(\mathrm{n}=49)$.

- All implants were stable at 5-year $(n=82)$ and 10-year $(n=49)$ follow-ups.

- No implant failures were reported.

Table 1 Implant positions

Maxilla

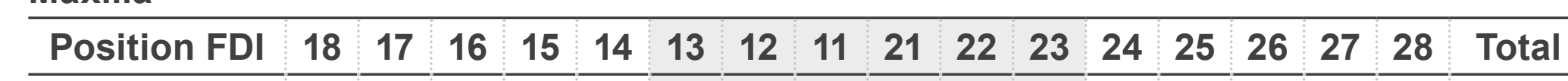

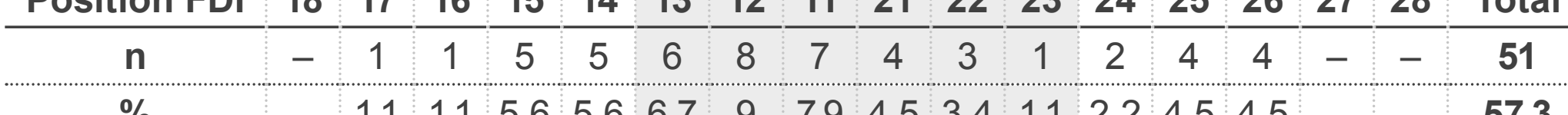

Mandible \begin{tabular}{c|c|c|c|c|c|c|c|c|c|c|c|c|c|c|c|c|c|}
\hline Position FDI & 48 & 47 & 46 & 45 & 44 & 43 & 42 & 41 & 31 & 32 & 33 & 34 & 35 & 36 & 37 & 38 & Total \\
\hline $\mathrm{n}$ & - & 2 & 4 & 6 & 4 & 1 & 3 & 3 & 2 & 2 & - & 2 & 4 & 5 & - & - & 38 \\
\hline
\end{tabular}

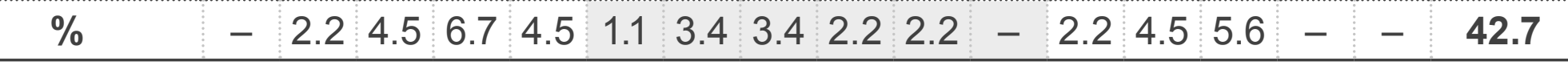
Four molar extraction sites received 2 implants each
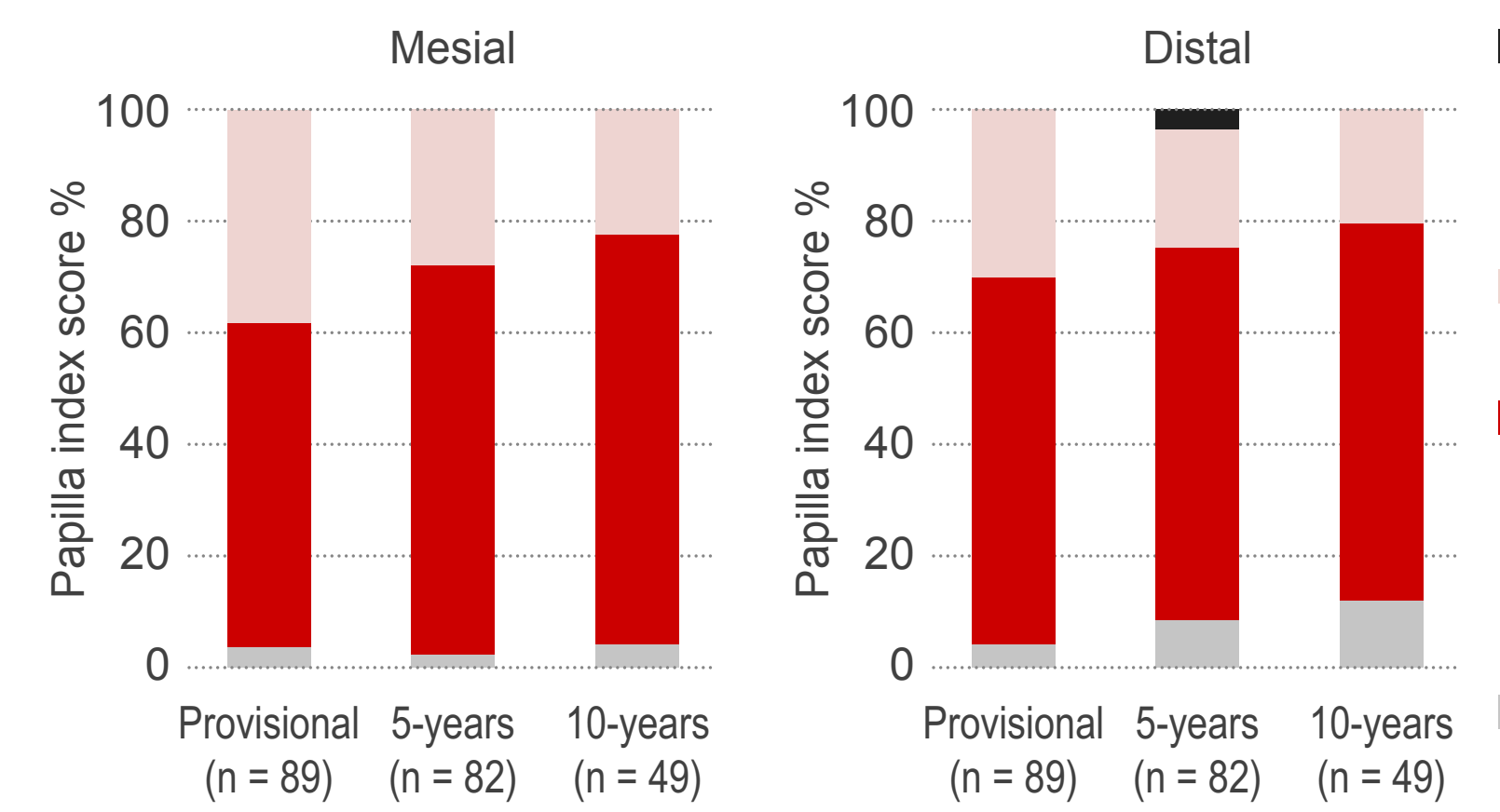

-PI 4 = Papilla is hyperplastic and covers too much and/or adjacent tooth.

$\mathrm{Pl} 3$ = Optimal soft tissue contour.

$P \mid 2=\geq 1 / 2$ of the height f the papilla is present, (n) between the teeth. betwenthe toul

$(\mathrm{n}=89) \quad(\mathrm{n}=82) \quad(\mathrm{n}=49)$ Provisional 5 -years 10 -years $\quad \mathrm{Pl} 1=<1 / 2$ of the height of the papilla is present. PI 0 = No papilla is present

\section{Clinical Case}

A 28 year-old female patient required replacement of her second molar (position 47). The tooth was carefully extracted and a Replace Select Tapered TiUnite WP $6.0 \times 13 \mathrm{~mm}$ implant was inserted with a torque of $45 \mathrm{Ncm}$. The implant was immediately loaded with a cement retained temporary crown. The final metal-ceramic crown was placed 3 months later. The patient's last follow-up was 11.98 years after implant placement.
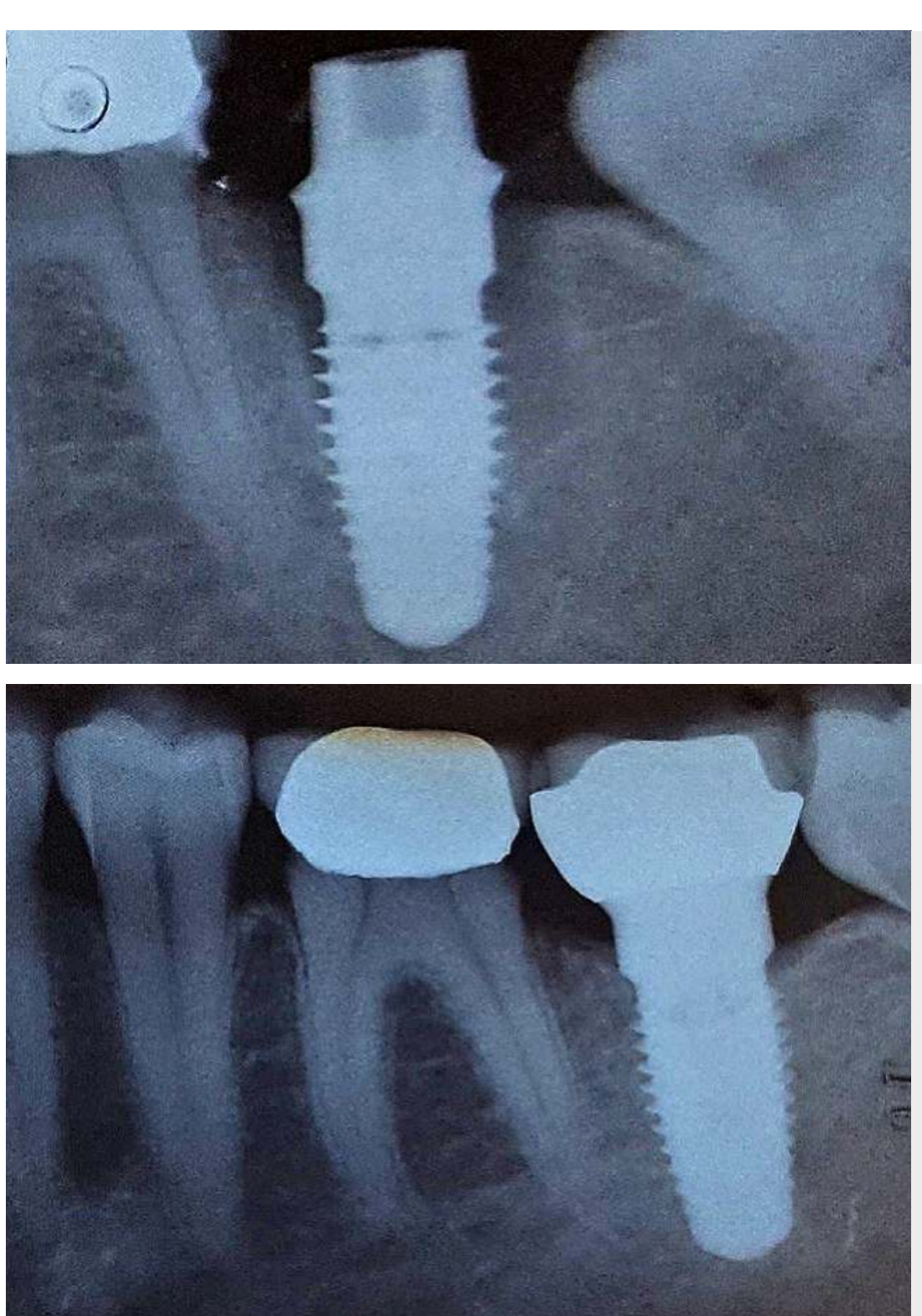

Figure 4 Radiograph at 3-year follow-up

Figure 3 Radiograph of implant at insertion into fresh extraction socket.

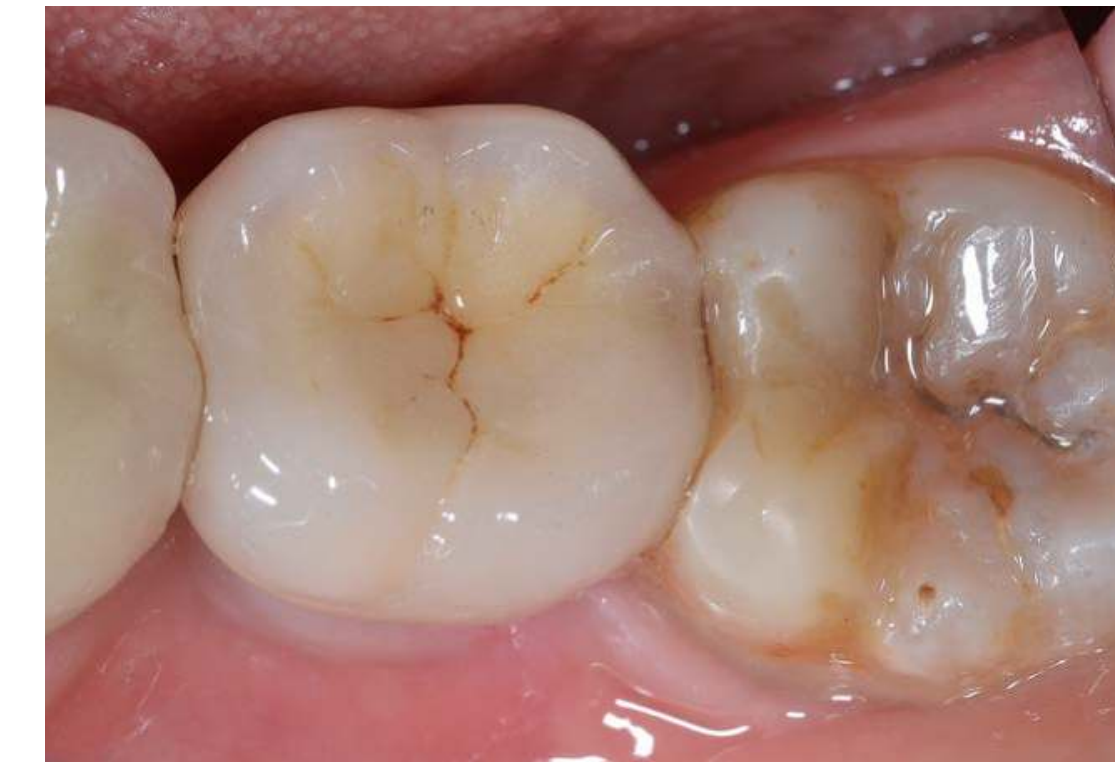

Figure 5 Occlusal view at las follow-up.

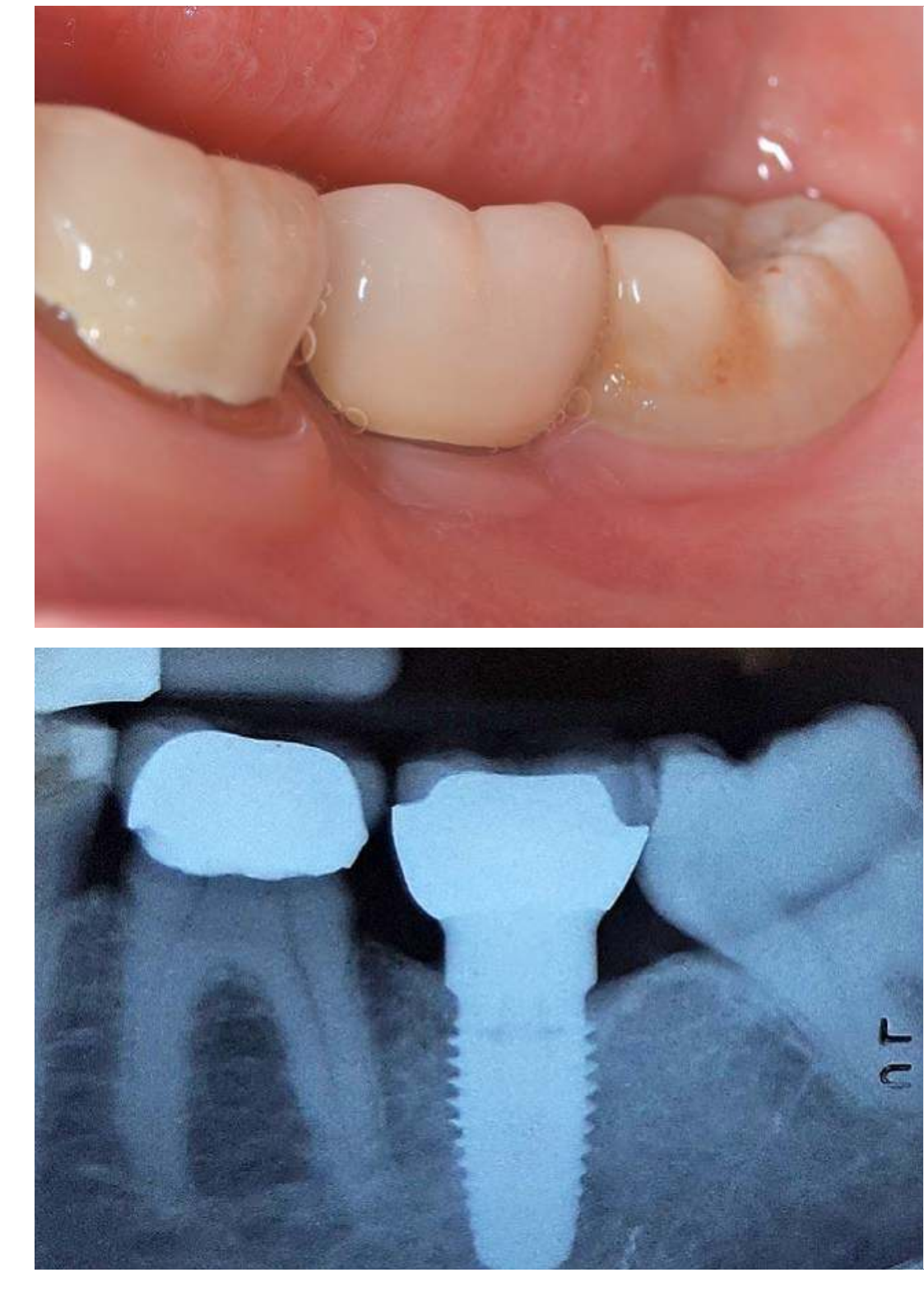

Figure 6 Lateral view at last follow-up.

Figure 7 Radiograph showing excellent bone levels at 11.98 years after insertion.

\section{Conclusions}

- Immediate and early loading of Replace Select Tapered TiUnite implants in fresh extraction sites yield very good treatment outcomes with regard to implan survival, soft-tissue health, and marginal bone response.

- This protocol can restore function quickly and effectively to patients with failing dentition.

\section{References}

Mura, P. (2012) Immediate loading of tapered implants placed in postextraction sockets: Retrospective analysis of the 5-year clinical outcome. Clin Implant Dent Relat Res 14:

Javed, F. \& Romanos, G. E. (2010) The role of primary stability for successful immediate loading of dental implants. A literature review. J Dent 38: 612-62

Chrcanovic, B. R., Martins, M. D. \& Wennerberg, A. (2015) Immediate placement of implants into infected sites: A systematic review. Clin Implant Dent Relat Res 17: e1-e16. Periontics Restortive Dent 17:326-333. 